\title{
Détection des oocystes de Cryptosporidium dans les fèces de caprins : comparaison entre un test d'agglutination au latex et trois autres techniques conventionnelles
}

\author{
Christophe Chartier ${ }^{\text {a* }}$, Marie-Pierre Mallereau-Pelleta, \\ Roselyne Mancassola ${ }^{\mathrm{b}}$, David NusSBAuM ${ }^{\mathrm{c}}$

\begin{abstract}
aAFSSA-Niort, Laboratoire d'Études et de Recherches Caprines, 60 rue de Pied de Fond, BP 3081, 79012 Niort Cedex, France

bINRA-Tours, Station de Pathologie Aviaire et de Parasitologie, 37380 Monnaie, France

${ }^{\mathrm{c}}$ VETOQUINOL Diagnostics, BP 189, 70204 Lure, France
\end{abstract}

(Reçu le 26 avril 2001 ; accepté le 28 septembre 2001)

\begin{abstract}
Detection of Cryptosporidium oocysts from goat kid faeces: comparison of a latex agglutination test with three other conventional techniques. A quantitative latex agglutination test (QLAT) with monoclonal antibodies for the detection of Cryptosporidium oocysts in faeces was compared with 3 other conventional techniques: Heine staining on faecal smears (HS) giving semi-quantitative results (scores from 1 to 5), sucrose flotation on diluted faeces (SF) with results expressed in oocysts/g of faeces (opg), direct ELISA (DE) giving qualitative results. Goat kid unconcentrated faecal samples (234) from 8 farms were processed according to the 4 techniques. Data were analyzed with Win Episcope 1.0 and Testview 1.1 softwares. The oocyst outputs ranged from 100000 (detection limit for SF) to 200 millions opg (mean: 15.2 millions opg). A very good agreement was recorded between QLAT and HS, SF, DE: Kappa values ranged between 0.82 and 0.90 . When considering the samples exhibiting oocysts (or not) as positive (or negative) using both HS and SF $(n=219)$, the sensitivity and specificity of QLAT were respectively 95.1 and $96.0 \%$. The lack of sensitivity was observed in faeces harboring a few oocysts $(\leq 200000 \mathrm{opg}$, scores $\leq 2)$ whereas the lack of specificity was only observed in 3 samples originating from the same farm. A significant correlation was calculated between the percentage of agglutination in QLAT and the number of oocysts in SF or scores in HS (Spearman correlation ranging from 0.45 to $0.48, p<0.001$ ). QLAT is a rapid, simple and reliable tool for routine detection of Cryptosporidium oocysts in faeces.
\end{abstract}

goat / Cryptosporidium / diagnosis / latex agglutination test

\footnotetext{
* Correspondance et tirés-à-part

Tél. : (33) 549796128 ; fax : (33) 549794219 ; e-mail : c.chartier@niort.afssa.fr
} 
Résumé - Un test quantitatif d'agglutination au latex (TQAL) à base d'anticorps monoclonaux pour la détection des oocystes de Cryptosporidium dans les fèces a été comparé à 3 autres techniques conventionnelles : coloration de Heine $(\mathrm{CH})$ sur frottis de fèces donnant un résultat semi-quantitatif (notes de 1 à 5), flottation au sucrose (FS) sur fèces diluées avec des résultats exprimés en oocystes par gramme de fèces (opg) et kit ELISA direct (ED) donnant des résultats qualitatifs. Des échantillons (234) de fèces de chevreaux issus de 8 fermes ont été étudiés selon les 4 techniques sans concentration préalable. Les données ont été analysées avec les logiciels Win Episcope 1.0 et Test-View 1.1. Les excrétions d'oocystes variaient de 100000 (seuil de détection pour la FS) à 200 millions d'oocystes par gramme de fèces (moyenne : 15,2 millions d'opg). Un très bon agrément a été noté entre TQAL et CH, FS, ED : les valeurs de Kappa variaient de 0,82 à 0,90 . Lorsque l'on considère comme positifs (ou négatifs) les échantillons présentant des oocystes (ou non) par les deux techniques FS et CH $(n=219)$, la sensibilité et la spécificité de TQAL étaient respectivement de 95,1 et 96,0\%. Le manque de sensibilité a été observé dans les fèces hébergeant peu d'oocystes $(\leq 200000 \mathrm{opg}$, notes $\leq 2)$ tandis que le manque de spécificité a été observé seulement dans 3 prélèvements issus de la même ferme. Une corrélation significative a été calculée entre le pourcentage d'agglutination dans TQAL et le nombre d'oocystes dans la FS ou les notes dans la $\mathrm{CH}$ (corrélation de Spearman variant de 0,45 à 0,48 ; $p<0,001)$. Le TQAL est une technique rapide, simple et fiable pour la détection en routine des oocystes de Cryptosporidium dans les fèces.

caprin / Cryptosporidium / diagnostic / test d'agglutination au latex

\section{INTRODUCTION}

Le diagnostic de la cryptosporidiose chez les jeunes ruminants fait appel à diverses techniques de mise en évidence des oocystes dans les matières fécales : étalement sur lame puis colorations, concentration par flottation ou sédimentation suivie éventuellement d'une coloration, méthodes immunologiques de type immunofluorescence ou immunoenzymatique (ELISA). La comparaison de ces différentes techniques donne les informations suivantes [1, 6-8, $10,13]$ :

- les techniques de coloration sont simples, peu onéreuses mais requièrent un œil exercé (relative subjectivité de la lecture pouvant entraîner des défauts de spécificité) et s'accompagnent d'une faible sensibilité et d'un résultat de type qualitatif. Dans le cas de la technique de Heine, l'utilisation d'un microscope à contraste de phase représente une contrainte supplémentaire.

- les techniques de concentration, comme la flottation au sucrose, sont plus sensibles et permettent une quantification des résultats. Les inconvénients sont là encore la difficulté de lecture et surtout la lourdeur de mise en œuvre (comptage des oocystes en cellule de Thoma).

- les techniques d'immuno-marquage, immunofluorescence et ELISA ont une haute sensibilité et une bonne spécificité. Pour l'ELISA, la technique est de surcroît « objective ». La réalisation de ces deux techniques reste cependant relativement lourde.

Une technique utilisant des microsphères de latex sensibilisées par un anticorps spécifique (Sphervet Crypto ${ }^{N D}$ ) a été développée récemment pour la cryptosporidiose ; des travaux préliminaires sur bovins se limitant à la comparaison de deux techniques immunologiques indiquent des caractéristiques de sensibilité et de spécificité comparables à celles de l'ELISA [11]. L'avantage de cette technique réside principalement dans sa simplicité et sa rapidité d'exécution, permettant de traiter aisément un grand nombre d'échantillons.

L'objectif de notre travail a ainsi été d'évaluer le test d'agglutination au latex sur des fèces de chevreaux infestés naturellement par Cryptosporidium sp. et présentant des concentrations variables en oocystes. Cette évaluation a été effectuée par rapport 
à 3 techniques conventionnelles dont l'une d'entre elles permettait le dénombrement des oocystes : la flottation quantitative au sucrose, la coloration semi-quantitative de Heine et un kit ELISA du commerce.

\section{MATERIEL ET METHODES}

\subsection{Choix des élevages et des prélèvements}

Une diversité dans l'origine des prélèvements a été recherchée. Ainsi, 8 élevages des départements des Deux-Sèvres et de la Vienne (France) présentant des cas de cryptosporidiose chez les chevreaux ont été retenus. L'échantillon de fèces $(n=234)$ a été récolté entre le 11/12/97 et le 16/02/98.

Afin d'obtenir des matières fécales présentant des concentrations très variables en oocystes de Cryptosporidium sp. [2], les prélèvements ont été réalisés sur des chevreaux âgés de 9-20 jours (prélèvements susceptibles d'être très riches en oocystes, $n=156$ ), des chevreaux âgés de $5-8$ jours ou âgés de plus de 3 semaines (prélèvements susceptibles d'être modérément riches en oocystes, $n=24$ ), des chevreaux âgés de moins de 5 jours ou des animaux adultes (prélèvements susceptibles d'être négatifs ou très pauvres en oocystes, $n=54$ ). Un échantillon correspond à un animal.

Les prélèvements ont été réalisés individuellement et aliquotés dès leur retour au laboratoire. Les différentes techniques ont été réalisées simultanément et en aveugle dans les 48 heures (conservation à $+4^{\circ} \mathrm{C}$ ).

La récolte des fèces a été réalisée à l'aide d'un sac plastique dans lequel l'animal passait ses quatre membres, le sac étant posé le soir et relevé le lendemain.

\subsection{Analyses des fèces}

La technique quantitative de flottation au sucrose utilisée a été celle décrite par Mancassola et al. [7] : 0,25 $\mathrm{g}$ de fèces ont été homogénéisés avec $750 \mu \mathrm{L}$ d'eau puis $4 \mathrm{~mL}$ de solution saturée au sucrose ont été rajoutés. Après agitation vigoureuse, les oocystes ont été comptés dans une cellule de Thoma. La sensibilité a été de 200000 oocystes par gramme de fèces (opg). Lors d'examen négatif, un contrôle a été réalisé directement entre lame et lamelle afin d'abaisser le seuil de sensibilité (par convention entre 1 et 200000 opg soit 100000 opg).

Dans la technique semi-quantitative de Heine [6], les fèces ont été mélangées à part égale $(3 \mu \mathrm{L})$ avec de la fuchsine de Ziehl puis étalées en couche mince. Immédiatement après séchage, la préparation a été recouverte d'huile d'immersion puis d'une lamelle et examinée à $\mathrm{G} \times 100$ à l'aide d'un microscope à contraste de phase. Le nombre d'oocystes par champ microscopique a été compté et une note de 0 à 5 a été attribuée : $0:$ absence d'oocystes ; $1:$ moins d'1 oocyste par champ ; 2 : 1-10 oocystes ; $3: 11-20$ oocystes ; $4: 21-30$ oocystes ; 5 : plus de 30 oocystes.

La technique ELISA réalisée a été celle indiquée par le fabricant (Pathasure Crypto ${ }^{\mathrm{ND}}$, Vétoquinol Diagnostics, Lure, France). Brièvement, à chaque puits de la microplaque préalablement recouvert par un anticorps monoclonal spécifique de C. parvum, C. muris et C. baileyi, deux gouttes de fèces diluées à tester ont été déposées et le tout a été laissé en incubation $30 \mathrm{~min}$ à température ambiante. Après cinq rinçages, le conjugué Cryptosporidium a été ajouté et la plaque a été laissée de nouveau $30 \mathrm{~min}$ à température ambiante. Après cinq rinçages, le substrat a été ajouté et la plaque a été laissée 15 min à température ambiante avant lecture visuelle. Une coloration bleue distincte a été considérée comme positive tandis qu'une absence de couleur ou une très faible teinte bleue a été considérée comme négative (témoins positif et négatif).

La technique d'agglutination au latex réalisée (Sphervet Crypto ${ }^{\mathrm{ND}}$ ) a été celle indiquée par le fabricant (Vétoquinol Diagnostics, Lure, France). Vingt microlitres 
de fèces préalablement dilués et centrifugés ont été placés dans les puits d'une microplaque. Quatre vingt microlitres de microsphères au latex de couleur jaune et sensibilisées par le même anticorps monoclonal que précédemment ont été rajoutés. La présence de $C$. parvum dans les fèces a provoqué l'agglutination de tout ou partie des sphères et ainsi une diminution de l'absorbance à la longueur d'onde $405 \mathrm{~nm}$. Après agitation de la plaque (15 secondes), l'absorbance a été lue une première fois (DO1) puis, après agitation pendant 15 minutes une seconde lecture a été faite (DO2). Le pourcentage d'agglutination a été calculé comme suit : \% agglutination $=[(1-\mathrm{DO} 2 / \mathrm{DO} 1) \times 100]$. Un échantillon a été défini comme positif lorsque l'agglutination était supérieure à $10 \%$.

\subsection{Analyse des données}

L'analyse des données a été classique : calcul des agréments par le coefficient Kappa variant de 0 (aucun agrément) à 1 (agrément parfait) pour les différents tests et évaluation de la sensibilité et de la spécificité par rapport à une technique de référence. Pour cette dernière, nous avons ainsi défini, comme échantillon positif ou négatif de référence, un échantillon de fèces qui présentait un résultat positif ou négatif aux 2 techniques parasitologiques simultanément en écartant de l'analyse tous les résultats discordants pour ces 2 techniques. Pour chaque comparaison de techniques 2 à 2 , un test de McNemar a été réalisé ; ce test ne prenant en compte que les réponses discordantes $(+-,-+)$, il a permis de dire si les différences observées étaient significatives $(P<0,05)$ ou non $(P>0,05)$. Les logiciels utilisés ont été Win Episcope 1.0 [4] et Testview 1.1 [5]. Les analyses statistiques non-paramétriques (corrélation des rangs de Spearman) ont été effectuées sur le logiciel Bidouille (INSERM) sur mini-ordinateur Vax.

\section{RESULTATS}

\subsection{Résultats généraux test par test}

Par la technique de flottation au sucrose, 153 examens ont été positifs, soit $65,4 \%$. Le niveau moyen d'excrétion pour les fèces positives a été de 15,2 millions d'oocystes par gramme de fèces avec un écart allant de 100000 à 200 millions d'opg (Fig. 1).

Avec la coloration de Heine, 151 prélèvements ont été positifs, soit $64,5 \%$. La notation moyenne pour les positifs (note de 1 à 5) a été de 3,44.

Avec le test ELISA, 137 prélèvements ont été positifs, soit $58,5 \%$. Suivant les recommandations du fabricant, la lecture s'est faite à l'œil pour déterminer le résultat. Toutefois, les densités optiques ont été enregistrées : la valeur moyenne est de 0,21 (écarts de 0,12 à 0,38 ) pour les résultats positifs et de 0,067 (écarts de 0,05 à 0,14 ) pour les résultats négatifs.

Avec le test d'agglutination au latex, 144 prélèvements ont été positifs, soit $61,5 \%$. Pour les prélèvements positifs, le pourcentage moyen d'agglutination était de

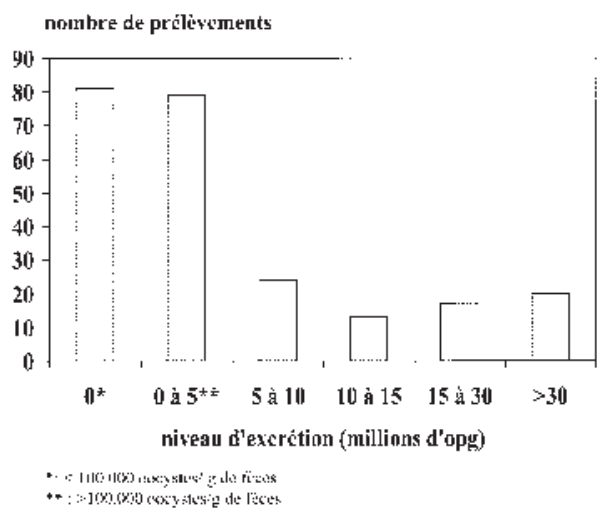

Figure 1. Distribution des prélèvements de fèces selon le niveau d'excrétion des oocystes (technique de flottation quantitative au sucrose). 
$49,8(11,1$ à 64,8$)$ tandis que pour les prélèvements négatifs il était de 1,67 $(0-9,8)$.

\subsection{Agrément entre les différentes techniques}

Le tableau I présente les différentes comparaisons des tests 2 à 2 : les valeurs de Kappa étaient toutes supérieures à 0,82 ce qui signifie un très bon agrément entre l'ensemble de ces tests. Par ailleurs, la valeur de t (table de l'écart-réduit) était supérieure à 12,6 ce qui indique une relation significative entre ces différents tests pris 2 à 2 $\left(P<10^{-9}\right)$. La comparaison des techniques par le test de McNemar a donné des résultats non significatifs sauf pour les comparaisons flottation au sucrose -ELISA et coloration de Heine-ELISA: dans ces 2 derniers cas le test ELISA a détecté un nombre significativement moins important de fèces positives en comparaison avec les 2 techniques parasitologiques.

De manière globale on a noté les plus fortes valeurs de Kappa entre les 2 techniques d'immunomarquage d'une part $(0,902$ entre agglutination au latex et ELISA) et les 2 techniques parasitologiques d'autre part $(0,86$ entre flottation au sucrose et coloration de Heine).

Tableau I. Valeurs de Kappa entre les différentes techniques de diagnostic (234 prélèvements).

\begin{tabular}{lccc}
\hline & $\begin{array}{c}\text { Flottation } \\
\text { au sucrose }\end{array}$ & $\begin{array}{c}\text { Coloration } \\
\text { de Heine }\end{array}$ & ELISA \\
\hline $\begin{array}{lccc}\text { Agglutination } \\
\text { au latex }\end{array}$ & 0,853 & 0,826 & 0,902 \\
Flottation au & - & 0,860 & NS \\
sucrose & & NS & $(p<047$ \\
$\begin{array}{l}\text { Coloration de } \\
\text { Heine }\end{array}$ & - & - & $0,001)$ \\
\hline
\end{tabular}

${ }^{a}$ Entre parenthèses figure le niveau de signification obtenu par le test de McNemar.

\section{3. Étude des discordances agglutination au latex/autres techniques}

\subsubsection{Comparaison agglutination au latex / flottation au sucrose}

La valeur du Kappa était de 0,853: 218 examens étaient concordants $(93,2 \%)$ et 16 étaient discordants $(6,8 \%)$. Les 16 examens discordants se décomposaient en :

- 12 examens négatifs en agglutination au latex et positifs en flottation au sucrose ;

-4 examens positifs en agglutination au latex et négatifs en flottation au sucrose.

Les 12 résultats négatifs en agglutination au latex présentaient des excrétions très faibles en flottation au sucrose : 150000 en moyenne et, par ailleurs, 5 de ces examens étaient également négatifs selon la technique de coloration de Heine.

A l'inverse, les 4 résultats positifs en agglutination au latex et négatifs en flottation au sucrose ont été confirmés négatifs en coloration de Heine et par la technique ELISA pour 3 d'entre eux. Cette discordance a porté sur 3 échantillons provenant d'un seul et même élevage (élevage 6). Par ailleurs, les pourcentages d'agglutination obtenus pour ces 3 échantillons en agglutination au latex étaient relativement faibles (13,1 à 19,3\%).

\subsubsection{Comparaison agglutination au latex / coloration de Heine}

La valeur du coefficient Kappa était de 0,826 : 215 prélèvements étaient concordants $(91,2 \%)$ et 19 discordants $(8,1 \%)$. Les prélèvements discordants se répartissaient comme suit :

- 13 examens positifs par la coloration de Heine et négatifs en agglutination au latex ;

-6 examens négatifs par la coloration de Heine et positifs en agglutination au latex.

Parmi les 13 résultats négatifs en agglutination au latex, 6 l'étaient également en flottation au sucrose, ce qui suggère un niveau 
d'excrétion très faible, voire nul (10 examens étaient d'ailleurs notés 1 en coloration de Heine).

Parmi les 6 résultats positifs en agglutination au latex, 3 d'entre eux étaient également trouvés positifs par la flottation au sucrose. Pour ces 3 examens, les pourcentages d'agglutination étaient supérieurs à $26 \%$ et l'un des 3 était également positif en ELISA. Les 3 autres échantillons sont les mêmes que ceux vus dans le chapitre précédent (élevage 6).

\subsubsection{Comparaison agglutination au latex / ELISA}

La valeur du Kappa était de 0,902 avec 11 examens discordants sur 234 réalisés (4,7\%).

- Neuf examens étaient positifs en agglutination au latex et négatifs en ELISA : parmi ces 9 examens, 6 étaient positifs en flottation au sucrose (3 à 100000 opg, 1 à 600000 opg, 1 à 1200000 opg) et 4 étaient positifs en coloration de Heine ( 3 notes à 2 , 1 note à 3). Dans 40 à $60 \%$ de ces cas le test ELISA a présenté un défaut de sensibilité. Dans 3 examens on a retrouvé le défaut de spécificité de l'agglutination au latex signalé auparavant (élevage 6).

- Deux examens étaient négatifs en agglutination au latex et positifs en ELISA : ces 2 examens étaient également positifs en flottation au sucrose (100 000 opg) et l'un d'entre eux était positif en Heine (note 1). Il s'agissait donc pour ces 2 cas d'un défaut de sensibilité de l'agglutination au latex (dont 1 avec un pourcentage d'agglutination à $9 \%$ ).

\subsubsection{Comparaison coloration de Heine / flottation au sucrose}

La comparaison coloration de Heine / flottation au sucrose a montré un Kappa de 0,860 avec un nombre d'examens discordants de 15 sur 234, soit 6,4\%. Huit examens étaient positifs en flottation au sucrose et négatifs en coloration de Heine tan- dis que 7 examens étaient négatifs en flottation au sucrose et positifs en coloration de Heine. Dans la majorité des cas, on se situait à la limite de détection pour les 2 techniques parasitologiques et, selon le cas, l'une ou l'autre s'avérait la plus sensible. Aucune supériorité d'un test par rapport à l'autre n'a pu être mise en évidence (test de McNemar non significatif).

\subsection{Spécificité et sensibilité du test d'agglutination au latex (Tab. II)}

Sur les 219 prélèvements positifs ou négatifs à la fois en flottation au sucrose et en coloration de Heine, la sensibilité de l'agglutination au latex était de 95,1\% (137/ $144)$ et sa spécificité de $96,0 \%(72 / 75)$. La valeur prédictive positive, c'est- à-dire la probabilité qu'un prélèvement déclaré positif l'ait été réellement, était de 137/140, soit $97,9 \%$. La valeur prédictive négative, probabilité qu'un prélèvement déclaré négatif l'ait été réellement, était de $72 / 79$, soit $91,1 \%$.

\subsection{Interprétation quantitative du test d'agglutination au latex et de l'ELISA}

Bien que l'interprétation des tests d'agglutination au latex et de l'ELISA soit de nature qualitative par rapport à un seuil de positivité $(10 \%$ d'agglutination pour le test d'agglutination au latex et seuil visuel pour

Tableau II. Caractéristiques du test d'agglutination au latex en prenant comme référence les résultats concordants de la flottation au sucrose et de la coloration de Heine.

\begin{tabular}{lccc}
\hline & \multicolumn{3}{c}{$\begin{array}{c}\text { Flottation au sucrose et coloration } \\
\text { de Heine }\end{array}$} \\
\cline { 2 - 4 } $\begin{array}{l}\text { Agglutination } \\
\text { au latex }\end{array}$ & Positives & Négatives & Total \\
\hline Positive & 137 & 3 & 140 \\
Négative & 7 & 72 & 79 \\
Total & 144 & 75 & 219 \\
\hline
\end{tabular}


Tableau III. Coefficients de corrélation de rang de Spearman entre les différentes techniques de diagnostic pour 144 examens positifs à la fois en flottation au sucrose et en coloration de Heine.

\begin{tabular}{lccc}
\hline & $\begin{array}{c}\text { Flottation } \\
\text { au sucrose }\end{array}$ & $\begin{array}{c}\text { Coloration } \\
\text { de Heine }\end{array}$ & ELISA \\
\hline $\begin{array}{l}\text { Agglutination } \\
\text { au latex }\end{array}$ & $0,48^{* * *}$ & $0,45^{* * *}$ & $0,43^{* * *}$ \\
$\begin{array}{l}\text { Flottation au } \\
\text { sucrose }\end{array}$ & - & $0,86^{* * *}$ & $0,61 * * *$ \\
$\begin{array}{l}\text { Coloration de } \\
\text { Heine }\end{array}$ & - & - & $0,50 * * *$ \\
\hline
\end{tabular}

$* * * P<0,001$

l'ELISA), il nous a semblé intéressant de voir si l'intensité de la réaction exprimée (\% d'agglutination et densité optique respectivement) était en relation avec le nombre d'oocystes présents évalué par flottation ou par coloration. Nous n'avons considéré que les prélèvements pour lesquels la réponse était positive simultanément par les 2 techniques parasitologiques, soit 144 analyses.

Les coefficients de corrélation de rang de Spearman figurent au tableau III. Quel que soit le couple de techniques considéré, ces valeurs oscillant de 0,43 à 0,86 étaient significatives $(p<0,001)$ indiquant une dépendance entre les variables prises 2 à 2 . Ces coefficients étaient cependant assez bas et homogènes pour l'agglutination au latex avec les 2 techniques parasitologiques $(0,45$ et 0,48$)$, plus élevées pour l'ELISA $(0,5$ et 0,61$)$ tandis qu'entre les 2 techniques parasitologiques la valeur du coefficient de Spearman était très forte $(0,86)$.

\section{DISCUSSION}

La mise en évidence des oocystes de cryptosporidies dans les matières fécales s'appuie sur des techniques de coloration, de concentration (sédimentation ou flottation) ou d'immuno-marquage. Les techniques de coloration comprennent principalement la coloration de Ziehl-Neelsen modifiée, la coloration de Kinyoun, la coloration auramine-phénol et la coloration négative de Heine [1]. Les avantages de ces techniques sont leur faible coût et leur rapidité de mise en œuvre tandis que les inconvénients résident dans la nécessité d'équipements spécifiques, microscope à fluorescence ou à contraste de phase pour les colorations auramine-phénol et Heine respectivement. En outre, la coloration de Ziehl-Neelsen, souvent considérée comme la technique de référence, peut s'accompagner d'un certain nombre de faux positifs (levures, spores de champignons ou de bactéries, globules gras) bien que la taille des éléments « pseudo-cryptosporidiens » permette théoriquement d'éviter la confusion [1]. Des défauts de sensibilité, par absence de coloration de certains oocystes, ont été également signalés en particulier avec les colorations « acid-fast» telles que Ziehl- Neelsen et Kinyoun [3]. Les techniques de concentration comprennent la flottation au sucrose et la sédimentation au formol-ether ou au formol-acétate d'éthyle. La flottation au sucrose [7] est une technique qui permet à la fois d'augmenter la sensibilité et de quantifier l'excrétion d'oocystes ce qui est très précieux lors d'essais thérapeutiques ou d'études épidémiologiques. Pour pallier la relative lourdeur de mise en œuvre de cette technique liée au comptage en cellule de Thoma, Naciri [9] a développé une variante rapide qualitative dans laquelle on mélange directement une goutte de fèces avec une goutte de solution au sucrose que l'on examine directement entre lame et lamelle.

Dans notre travail les résultats obtenus par examen direct (technique de Heine) ou après flottation au sucrose étaient similaires : une excellente concordance a été notée et il était difficile de conclure à un défaut de sensibilité d'une technique plutôt que de l'autre. Ces données sont en apparente contradiction avec celles de Weber et al. [13] et de Webster et al. [14] pour qui les techniques de concentration permettent de détecter 7 à 20 fois plus d'éléments parasitaires. En fait, 
ces derniers auteurs associent une technique de flottation au sucrose à une mise en évidence des oocystes par coloration à l'auramine-phénol ou en immuno-fluorescence. Par ailleurs, la sensibilité d'une coloration sur étalement des fèces dépend bien évidemment du nombre de champs microscopiques examinés et peut donc être notablement améliorée.

Les techniques de marquage immunologique s'appuient sur l'utilisation d'anticorps monoclonaux conjugués à des marqueurs fluorescents, des enzymes (ELISA) ou d'autres supports (microsphères de latex).

Les techniques ELISA ont été comparées aux techniques conventionnelles (concentration et/ou coloration) par différents auteurs. Pour Robert et al. [12], la technique ELISA montre une sensibilité équivalente par rapport à la flottation avec $96 \%$ d'agrément. Les données de Newman et al. [10] font état d'un déficit de sensibilité de la technique ELISA par rapport à une sédi mentation au formol-acétate d'éthyle suivie d'une coloration de Ziehl-Neelseen. À l'inverse, McCluskey et al. [8] ont démontré une concordance de seulement $72 \%$ entre la technique ELISA et la coloration de Kinyoun sur frottis non enrichi (Kappa = 0,42 ), la technique ELISA permettant de détecter plus de fèces positives. Pour ces derniers auteurs, cette meilleure sensibilité pourrait être en rapport avec l'aptitude de l'ELISA à détecter des fragments d'oocystes ce que ne permet pas la technique de coloration. Nos propres données ont montré une excellente concordance de l'ELISA avec les 2 techniques parasitologiques $($ Kappa $=0,84)$ avec toutefois une sensibilité un peu plus faible de l'ELISA $(93,1 \%)$.

Le test d'agglutination au latex n'a, à notre connaissance, fait l'objet que d'une seule évaluation [11]. En considérant l'ELISA comme test de référence, Nussbaum et al. [11] ont montré que le test d'agglutination au latex présentait une sensibilité de
$97,9 \%$ et une spécificité de $97,4 \%$. Dans notre travail, la comparaison de l'agglutination au latex avec l'ELISA a montré également un très bon agrément des deux techniques (Kappa $=0,90 ; 95,3 \%$ d'examens concordants). L'agrément de l'agglutination au latex avec les techniques parasitologiques est un peu moins élevé (Kappa entre 0,83 et 0,85 ) en raison de quelques défaillances de sensibilité sur des matières fécales pauvres en oocystes. Ainsi, la sensibilité maximale de l'agglutination au latex a pu être évaluée à 95,1\%. Cette valeur est probablement légèrement surestimée car, dans la définition de notre technique de référence (" gold standard»), nous n'avons retenu que les résultats doublement positifs (ou négatifs) par les techniques parasitologiques écartant du même coup les prélèvements plus pauvres sur lesquels précisément la sensibilité de l'agglutination au latex aurait été intéressante à mesurer. Cette valeur de sensibilité relativement basse peut en partie s'expliquer par la grande maîtrise des 2 techniques parasitologiques, coloration de Heine et flottation au sucrose, qui ont été pratiquées en routine par les auteurs depuis de nombreuses années. Incontestablement la sensibilité de ces 2 techniques parasitologiques s'en est trouvée amplifiée et cette situation n'est peut-être pas celle rencontrée dans la majorité des laboratoires de diagnostic vétérinaire. L'excrétion moyenne d'oocystes lors de cryptosporidiose aiguë tant chez le chevreau que chez le veau [15] étant de plusieurs millions par gramme de fèces, les différences de sensibilité relevées dans ce travail entre les tests ne sont pas préjudiciables à l'activité diagnostique. Les 4 résultats faussement positifs trouvés dans ce travail peuvent correspondre à un réel déficit de spécificité (96\%) sur quelques échantillons mais pourraient également être liés à la détection de fragments d'oocystes non retrouvés par les techniques de coloration ou de flottation [8]. Cette dernière hypothèse 
n'est cependant pas supportée par les résultats en ELISA qui sont négatifs pour 3 de ces échantillons.

Le pourcentage d'agglutination des sphères étant proportionnel à la quantité d'antigène présent dans l'échantillon, il semblait intéressant de vérifier si l'agglutination au latex pouvait être utilisée de manière quantitative. Le coefficient de corrélation des rangs de Spearman entre le pourcentage d'agglutination et la quantité d'oocystes par gramme de fèces en flottation était en effet significatif $(P<0,001)$. Le pourcentage d'agglutination a ainsi une valeur indicative de la quantité d'oocystes présents dans les matières fécales mais ne permet pas une utilisation directe à des fins diagnostiques en raison de la valeur modérée du coefficient de corrélation $(<0,48)$.

Le test d'agglutination au latex présente une très bonne concordance globale avec les 3 autres techniques de diagnostic : flottation au sucrose, coloration de Heine et ELISA. La facilité d'exécution autorisant la réalisation simultanée de plusieurs analyses, la rapidité d'obtention du résultat (20 minutes environ) et l'objectivité de la lecture permettent en outre le traitement aisé et fiable d'un grand nombre de prélèvements (série de quinze à la fois).

\section{RÉFÉRENCES}

[1] Casemore D.P., Laboratory methods for diagnosing cryptosporidiosis, J. Clin. Pathol. 44 (1991) 445-451.

[2] Chartier C., Mallereau M.-P., Lenfant D., Efficacité du lactate d'halofuginone dans la prévention de la cryptosporidiose chez le chevreau nouveau-né, Rev. Méd. Vét. 150 (1999) 341-348.

[3] Fayer R., Cryptosporidiosis: current strategies to meet a widespread problem, in: Proceedings of the VIth Int. Coccidiosis Conf., Guelph, Ontario, Canada, 1993, 95-101.

[4] Frankena K., Noordhuizen J.P., Willenberg P., Voorthuyzen P.F., Goelema J.O., Episcope: computer programs in veterinary epidemiology, Vet. Rec. 126 (1990) 573-576.

[5] Gardner I.A., Holmes J.C., Testview : a spreadsheet program for evaluation and interpretation of diagnostic tests, Prev. Vet. Med. 17 (1993) 9-18.

[6] Heine J., Eine einfache Nachweismethode für Kryptosporidien in Kot, Zentralbl. Veterinaermed. Reihe B 29 (1982) 324-327.

[7] Mancassola R., Repérant J.M., Naciri M., Chartier C., Chemoprophylaxis of Cryptosporidium parvum infection with paromomycin in kids and immunological study, Antimicrob. Agents Chemother. 39 (1995) 75-78

[8] McCluskey B.J., Greiner E.C., Donovan G.A., Patterns of Cryptosporidium oocyst shedding in calves and a comparison of two diagnostic methods, Vet. Parasitol. 60 (1995) 185-190.

[9] Naciri M., Cryptosporidiose des ruminants et santé publique, Point Vét. 26 (1994) 875-881.

[10] Newman R.D., Jaeger K.L., Wuhib T., Lima A.A.M., Guerrant R.L., Sears C.L., Evaluation of an antigen capture enzyme-linked immunosorbent assay for detection of Cryptosporidium oocysts, J. Clin. Microbiol. 31 (1993) 2080-2084.

[11] Nussbaum D., Salord J.R., Nahama A.G., Rimmele D.D., Evaluation of three antigenic detection tests for rotavirus, E. coli $\mathrm{K} 99$ and Cryptosporidiumparvum in bovine faeces, J. Vet. Diagn. Invest. 11 (1999) 314-318.

[12] Robert B., Ginter A., Antoine H., Collard A., Coppe B., Diagnosis of bovine cryptosporidiosis by an enzyme-linked immunosorbent assay, Vet. Parasitol. 37 (1990) 1-8

[13] Weber R., Bryan R.T., Bishop H.S., Wahlquist S.P., Sullivan J.J., Juranek D.D., Threshold of detection of Cryptosporidium oocysts in human stool specimens: evidence for low sensitivity in current diagnostic methods, J. Clin. Microbiol. 29 (1991) 1323-1327.

[14] Webster K.A., Smith H.V., Giles M., Dawson L., Robertson L.J., Detection of Cryptosporidium parvum oocysts in faeces: comparison of conventional coproscopical methods and the polymerase chain reaction, Vet. Parasitol. 61 (1996) 5-13.

[15] Xiao L., Herd R.P., Infection patterns of Cryptosporidium and Giardia in calves, Vet. Parasitol. 55 (1994) 257-262. 